\title{
ESTIMACIÓN DE LA RECAUDACIÓN POTENCIAL EN EL IMPUESTO AL TRABAJO Y A LOS INGRESOS AL CAPITAL: COMPARATIVO ENTRE MÉXICO Y ESTADOS UNIDOS
}

\author{
Francisco Beltrán Silva* \\ Instituto Tecnológico Autónomo de México \\ (Recibido 16 agosto de 2013, aceptado 18 de noviembre de 2013)
}

\begin{abstract}
Resumen
El presente documento argumenta, mediante la estimación de un modelo de equilibrio general y un análisis de largo plazo, que tanto México como Estados Unidos se encuentran lejos del rango prohibitivo en las curvas de Laffer para el impuesto al trabajo y al capital. Sin embargo, en términos de la montaña de Laffer, el análogo de la curva de Laffer en más de dos dimensiones, variando de forma conjunta ambos impuestos se obtiene que ambos países podrían aumentar más la recaudación en el largo plazo disminuyendo el impuesto al capital y aumentando el impuesto al trabajo, que elevando ambos.
\end{abstract}

\section{Abstract}

This paper argues, by estimating a general equilibrium model and a long-term analysis, that Mexico and the United States are far from the prohibitive range of the Laffer curves for both the tax on labor and that on capital. However, analyzing the variations of these two taxes jointly in terms of the Laffer hill, the analogue of the Laffer curve in more than two dimensions, it can be concluded that both countries could raise more revenue in the long run by reducing the tax on capital and increasing the tax on labor than by raising both.

Clasificación JEL: D50, E20, E13, H20.

Palabras clave: Equilibrio General, Impuestos, Curva de Laffer.

* Instituto Tecnológico Autónomo de México (ITAM). Río Hondo No. 1, Col. Progreso Tizapán, México, D.F., México, C.P. 01080 Tel. +52 (55) 5628 4000. Correo electrónico: bs.franco@gmail.com 


\section{Introducción}

En algunos círculos empresariales y políticos existe la opinión de que en México la carga tributaria es muy alta, en particular que las tasas impositivas son muy elevadas. Sin embargo, las tasas efectivas, es decir, la recaudación obtenida por concepto de distintos impuestos como proporción de las bases impositivas respectivas, son bajas. Las tasas efectivas son distintas de las legalmente impuestas debido, entre otras razones, a evasión y elusión fiscal, deducciones, fallas en la administración tributaria, así como regímenes y tratamientos especiales.

El presente documento, que constituye una extensión a Uhlig y Trabandt (2009), argumenta, mediante la estimación de un modelo de equilibrio general y un análisis de largo plazo, que México se encuentra muy lejos del rango prohibitivo en las curvas de Laffer para el impuesto al trabajo y al capital, rango donde un aumento en la tasa respectiva disminuye la recaudación. Tanto para México como para Estados Unidos se encuentra que actualmente los recortes de impuestos en el trabajo y el capital no generarían el crecimiento suficiente en la recaudación por el aumento de la base para compensar las pérdidas en recaudación asociadas a la baja en la tasa.

El presente estudio arroja resultados similares a los de Uhlig y Trabandt (2009) para Estados Unidos, la contribución de este documento es replicar dicho análisis para el caso de México, los resultados para Estados Unidos varían con respecto a los presentados en Uhlig y Trabandt (2009) debido principalmente al cambio en el periodo de estudio. El periodo de análisis tanto para México como para Estados Unidos es de 2003 a 2010.

En el documento se analiza la forma en que se ajusta la recaudación agregada ante cambios en los impuestos al trabajo y a los ingresos al capital, comparando la evolución de las sendas de crecimiento balanceado en un modelo de crecimiento neoclásico al variar las tasas impositivas. Para poder llevar a cabo este análisis se estiman tasas impositivas efectivas para el trabajo, el capital y el consumo siguiendo la metodología establecida por Mendoza et al. (1994). Las tasas efectivas calculadas con esta metodología son una buena estimación del efecto que tienen las tasas impositivas estatutarias sobre la economía agregada y son útiles para modelos de equilibrio general dinámicos con agente representativo.

Mediante este ejercicio se busca estimar la posición en que se encuentran México y Estados Unidos con respecto a su recaudación potencial en el impuesto al trabajo y a los ingresos al capital. Las diferencias se explican principalmente por el nivel de las tasas impositivas efectivas. Aunque los resultados de este estudio arrojen que tanto Estados Unidos como México se encuentran en posibilidades de aumentar la recaudación mediante alzas en las tasas impositivas eso no significa que deban aumentarlas. Llegar a un máximo recaudatorio no debe ser un fin del gobierno, los impuestos en general distorsionan la actividad económica y provocan que la asignación de recursos sea menos eficiente.

El documento se estructura de la siguiente forma: en la sección 2 se presenta una breve revisión de literatura referente a la curva y montaña de Laffer; en la sección 3 se describe y soluciona el modelo de equilibrio general empleado, en 
esta sección se obtienen las ecuaciones necesarias para los cálculos numéricos; en la sección 4 se explica la forma en la que se calibra el modelo y se presentan las estimaciones de las tasas impositivas efectivas; en la sección 5 se presentan las estimaciones de las curvas de Laffer en el impuesto al trabajo y a los ingresos al capital para Estados Unidos y México; en la sección 6 se explica cómo se genera la montaña de Laffer y se presenta una estimación para ambos países, en la sección 7 se presenta un resumen de las estimaciones más importantes y en la sección 8 se presentan las conclusiones.

\section{Curva y Montaña de Laffer}

En general, los impuestos per se desincentivan la actividad económica y con ello la base impositiva sobre la que actúan. Lo que dice la teoría de la curva de Laffer es que, para niveles bajos en la tasa impositiva, incrementos en la misma generan aumentos en la recaudación agregada y el aumento en la tasa impositiva domina la caída en la base recaudatoria. A partir de un cierto nivel, aumentos en la tasa impositiva en cuestión provocan caídas en la recaudación, pues el aumento en la tasa no compensa la caída en la base impositiva, ver Laffer (1981).

En términos generales la curva de Laffer describe la relación mencionada entre la tasa de un impuesto en particular y la recaudación agregada. Las funciones para el impuesto al trabajo y al capital se pueden representar de la siguiente manera:

$$
T_{t}=f\left(\tau^{n}\right) \text { y } T_{t}=f\left(\tau^{k}\right),
$$

donde $\tau^{n}$ y $\tau^{k}$ representan las tasas de los impuestos al trabajo y a los ingresos al capital, y $T_{t}$ es la recaudación agregada del sistema impositivo en conjunto.

La respuesta de la recaudación agregada ante variaciones conjuntas de ambos impuestos se puede apreciar a través de una montaña de Laffer. La montaña de Laffer describe la relación que existe entre la recaudación agregada y distintas combinaciones de impuestos al trabajo y al capital, permitiendo ver la relación. Ésta se puede representar como una función que depende de dos tasas impositivas, en este caso el impuesto al trabajo y a los ingresos al capital:

$$
T_{t}=f\left(\tau^{k}, \tau^{n}\right) .
$$

Para cada nivel recaudatorio, existen varias combinaciones de tasas impositivas efectivas que dan lugar a una misma recaudación. Al conjunto de combinaciones de tasas que dan lugar a una misma recaudación se le conoce como curva de iso-recaudación; podemos encontrar una curva de iso-recaudación para cada nivel recaudatorio, ver Laffer (1981). Una curva de iso-recaudación, se puede dibujar como un conjunto de coordenadas en un plano de dos dimensiones, donde el eje horizontal representa el impuesto al trabajo y el eje vertical el impuesto al capital. ${ }^{1}$

\footnotetext{
1 La forma de las curvas en la Gráfica 1 es arbitraria, la forma de éstas dependerá de las particularidades de la economía en cuestión.
} 
Gráfico 1. Curva de iso-recaudación

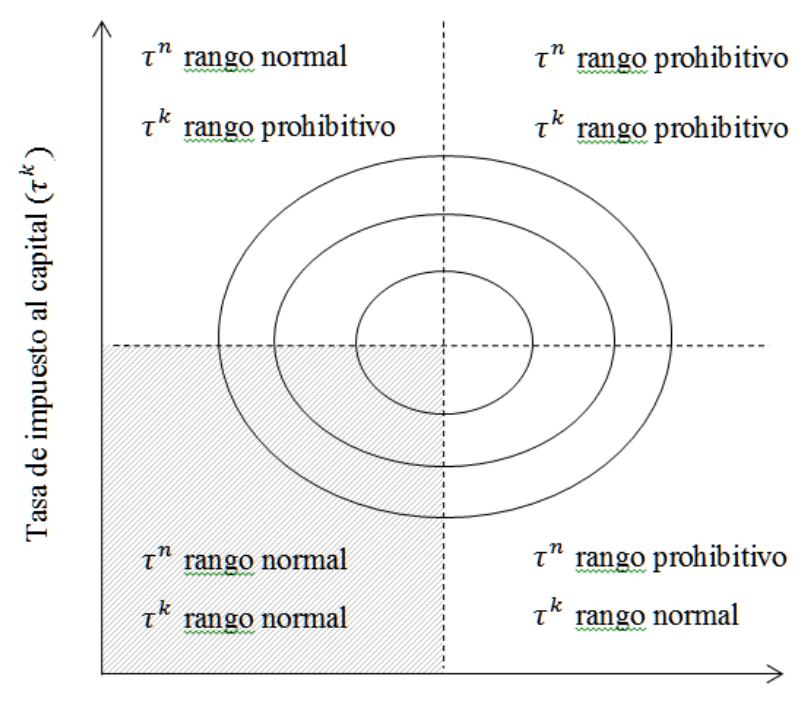

Tasa de impuesto al trabajo $\left(\tau^{n}\right)$

Fuente: Elaborado con base en Laffer (1981).

En la Gráfica 1 se puede ver que en cualquier otra región diferente a la normal (la que presenta el sombreado), una disminución en cualquiera de las tasas puede ir acompañada de la disminución en la otra, sin implicar una disminución en la recaudación total. Sin embargo, para mantener la recaudación constante en la región mencionada una disminución en una tasa tiene que ir acompañada por el aumento en otra.

La forma de representar una recaudación más alta en este diagrama es moviéndonos hacia una elipse con un área más pequeña. De aquí podemos observar también que mientras mayor sea la recaudación requerida, el conjunto de tasas que resultan en dicha recaudación es más reducido; existe, por lo tanto, un punto máximo tal que la recaudación no puede aumentar variando estos dos impuestos, de esta lógica se deriva el término montaña de Laffer.

Al igual que con las curvas de Laffer para cada impuesto, el punto en donde se encuentra una economía dentro de la montaña de Laffer depende del nivel actual de las tasas y del valor de las elasticidades de la oferta y demanda de los factores empleados en la producción. Determinar esta posición depende, entre otras cosas, de las estimaciones de las elasticidades. 


\section{Descripción del modelo}

Siguiendo a Uhlig y Trabandt (2009), se emplea un modelo de equilibrio general dinámico donde el tiempo es discreto e infinito, $t=1,2, \cdots, \infty$. El hogar representativo maximiza la suma de los flujos de utilidad en valor presente a lo largo de su vida, sujeto a una restricción presupuestal intertemporal y una ecuación de acumulación de capital.

Formalmente el hogar resuelve el siguiente problema:

$$
\max _{\left(c_{t}, n_{t}, k_{t}, x_{t}, b_{t}\right)}=E_{0} \sum_{(t=0)}^{\infty} \beta^{t}\left[u\left(c_{t}, n_{t}\right)+\nu\left(g_{t}\right)\right]
$$

sujeto a

$$
\begin{gathered}
\left(1+\tau_{t}^{c}\right) c_{t}+x_{t}+b_{t}=\left(1-\tau_{t}^{n}\right) w_{t} n_{t}+\left(1-\tau_{t}^{k}\right)\left(d_{t}-\delta\right) k_{t-1}+ \\
\delta k_{t-1}+R_{t}^{b} b_{t-1}+s_{t}+\Pi_{t}+m_{t} \\
k_{t}=(1-\delta) k_{t-1}+x_{t} .
\end{gathered}
$$

Los hogares toman el consumo de gobierno como dado y éste les brinda utilidad. Los hogares reciben ingresos por: salarios $w_{t}$, dividendos $d_{t} \mathrm{y}$ utilidades $\Pi_{t}$ provenientes de las empresas, un flujo exógeno $m_{t}$ por la posesión de activos, transferencias de suma fija $s_{t}$ por parte del gobierno e intereses $R_{t}^{b}$ por la posesión de bonos de deuda del gobierno.

Los hogares son propietarios del capital, se lo alquilan a las empresas y reciben dividendos netos de depreciación. Los hogares pueden emplear estos ingresos en consumo $c_{t}$, ahorro $b_{t}$ e inversiones de capital $x_{t}$. En esta economía simplificada se pagan impuestos sobre el consumo $\tau_{t}^{c}$ y sobre los ingresos por trabajo $\tau_{t}^{n}$ y capital $\tau_{t}^{k}$. Los impuestos a los ingresos al capital se aplican sobre los dividendos netos de depreciación.

Como en Uhlig y Trabandt (2009), se permite que los flujos $m_{t}$ sean negativos y por lo tanto que los activos sean obligaciones. Esto permite capturar una balanza comercial positiva o negativa, donde $m_{t}$ son las importaciones netas, introduciendo el comercio internacional en una forma muy simple.

Esto hace que en la senda de crecimiento balanceado el modelo sea consistente con una economía abierta. La balanza comercial influirá en la reacción de estado estacionario del factor trabajo a cambios en los impuestos y por lo tanto afectará la forma de la curva de Laffer.

La empresa representativa maximiza sus ganancias:

$$
\max _{k_{t-1}, n_{t}} y_{t}-d_{t} k_{t-1}-w_{t} n_{t}
$$

donde

$$
y_{t}=\xi^{t} k_{t-1}{ }^{\theta} n_{t}{ }^{1-\theta} \quad \text { y } \xi=\psi^{1-\theta} .
$$

De este modo $y_{t}$ representa la producción y el producto interno bruto de la economía, $\xi^{t}$ es exógena y denota la tendencia de la productividad total de 
los factores. Mientras que $\psi$ representa el factor de crecimiento de la economía y $\theta$ la elasticidad del producto con respecto al capital.

El gobierno enfrenta una restricción presupuestal de la forma:

$$
g_{t}+s_{t}+R_{t}^{b} b_{(t-1)}=b_{t}+T_{t},
$$

donde la recaudación está dada por

$$
T_{t}=\tau_{t}^{c} c_{t}+\tau_{t}^{n} w_{t} n_{t}+\tau_{t}^{k}\left(d_{t}-\delta\right) k_{t-1} .
$$

Se asume que los flujos provenientes del activo exógeno, la deuda y el gasto crecen de acuerdo al factor de crecimiento de la producción, por lo que la deuda gubernamental y el gasto no se desvían de sus sendas de crecimiento balanceado, esto implica la relación siguiente:

$$
m_{t}=\psi^{t} \bar{m} b_{t-1}=\psi^{t} \bar{b} g_{t}=\psi^{t} \bar{g} .
$$

Cuando cambian las tasas impositivas la recaudación varía y las transferencias gubernamentales se ajustan de acuerdo a la restricción presupuestal, esto se resume en la siguiente ecuación:

$$
s_{t}=\psi^{t} \bar{b}\left(\psi-R_{t}^{b}\right)+T_{t}-\psi^{t} \bar{g} .
$$

La elasticidad Frisch es la elasticidad de la oferta laboral con respecto al salario manteniendo la utilidad marginal del ingreso constante, ésta es igual a $\varphi$. La función de utilidad empleada se caracteriza por presentar una elasticidad Frisch constante, que no varía con el nivel de consumo o trabajo:

$$
\begin{gathered}
u(c, n)=\frac{1}{1-\eta}\left(c^{1-\eta}\left(1-\kappa(1-\eta) n^{1+\frac{1}{\varphi}}\right)^{\eta}-1\right) \\
\eta>0 \quad \eta \neq 1 \quad \kappa>0 \\
u(c, n)=\log (c)+\kappa n^{1+\frac{1}{\varphi}} \\
\eta=1 \quad \kappa>0
\end{gathered}
$$

Esta función presenta una elasticidad de sustitución intertemporal constante igual a $\frac{1}{\eta}$ y es consistente con el crecimiento de largo plazo, ver Uhlig y Trabandt (2009).

En equilibrio, los hogares escogen asignaciones que maximizan su utilidad y las empresas sus ganancias. El gobierno establece políticas tales que satisfacen su restricción presupuestal. Se escogen las relaciones o ecuaciones claves que resultan de resolver el modelo y que son necesarias para calcular las curvas de Laffer. El crecimiento balanceado después de impuestos de cualquier activo en esta economía es $R=\frac{\psi^{\eta}}{\beta}$. Todas las variables excepto las horas trabajadas, las tasas de interés y los impuestos, crecen a una tasa constante de acuerdo con el factor de crecimiento de la producción.

Resolviendo el problema de equilibrio general a lo largo de la senda de crecimiento balanceado y con algo de álgebra se obtiene una ecuación no lineal en $\bar{n}$ que se puede resolver numéricamente dados los valores para los parámetros 
en las funciones de producción y utilidad, las tasas impositivas efectivas y los niveles para $\bar{m}, \bar{g}$ y $\bar{b}$. De acuerdo con Uhlig y Trabandt (2009) si $\bar{m} \leq \bar{g}$ entonces la solución para $\bar{n}$ es única. Dicha ecuación es la siguiente:

$$
0=\left(\kappa\left(\eta\left(1-\frac{\bar{x}}{y}+\frac{\left(m_{t}-g_{t}\right)}{\frac{y_{t}}{\bar{n}}}\left(\frac{1}{\bar{n}}\right)\right)\left(\left(\frac{1+\tau_{t}^{c}}{1-\tau_{t}^{n}}\right)\left(\frac{1+\frac{1}{\varphi}}{1-\theta}\right)\right)-\eta+1\right)\right)^{-\frac{\varphi}{1+\varphi}}-\bar{n}
$$

A partir de esta ecuación y de otras relaciones derivadas de la solución del modelo de equilibrio general así como de los niveles en los parámetros para Estados Unidos y México, se obtienen las curvas de Laffer y la correspondiente montaña variando las tasas impositivas correspondientes para cada uno de los países. Para llevar a cabo las estimaciones y los cálculos numéricos para cada país, es necesario calcular ciertos agregados macroeconómicos como porcentaje del producto interno bruto y las tasas efectivas de impuestos que permitan ajustar el modelo a ambos países.

\section{Calibración y parametrización}

Se calibró el modelo con datos para Estados Unidos y México del 2003 al 2010. Se utilizan datos de Annual Macro-Economic Database (AMECO), de la Comisión Europea, de la base de datos de la OCDE y estadísticas de la Secretaría de Hacienda y Crédito Público. Para el presente documento, al igual que en Uhlig y Trabandt (2009), se utiliza la metodología de Mendoza et al. (1994) para calcular las tasas impositivas efectivas promedio a partir de cuentas nacionales y de recaudación tanto para Estados Unidos como para México.

La medida para el nivel de deuda gubernamental para ambos países es la deuda total del gobierno central como porcentaje del producto interno bruto (PIB), provista por la OCDE.

Las horas trabajadas para el modelo se calculan de la siguiente forma, se toma el total de horas trabajadas al año, se divide por la población de entre 15 y 64 años y el resultado se divide entre lo que resulta de multiplicar 365 por 14, donde 365 son los días del año y 14 son las horas diarias que se pueden asignar a trabajo y ocio, ver Ragan (2005).

Varios de los parámetros, como en Uhlig y Trabandt (2009), son estándares. Para la elasticidad de sustitución intertemporal se emplea un valor de 0.5, en línea con Uhligh y Trabandt (2009). Para el valor de la elasticidad Frisch de la oferta de trabajo se emplea el valor de 1 en línea con Uhlig y Trabandt (2009). La participación del capital en la producción se asume en 0.38 , por lo que la participación del trabajo es de 0.62. La tasa de depreciación se asume en 0.07. Para el factor de crecimiento se utiliza el valor del crecimiento anual del PIB per cápita.

Los parámetros que caracterizan la producción y las preferencias se mantienen para ambos países, de modo que las disparidades entre México y Estados Unidos surjan de diferencias en política fiscal y agregados macroeconómicos. Dado un nivel de trabajo inicial, se obtiene una solución para $\kappa$ consistente con los demás parámetros iniciales, donde $\kappa$ es una medida para la preferencia por el ocio. En términos generales para calibrar se utilizan los promedios, durante el periodo comprendido entre 2003 y 2010, de las observaciones en las siguientes variables: 
Tabla 1. Variables para calibrar: México y Estados Unidos

\begin{tabular}{|c|c|c|c|c|c|c|c|c|}
\hline$\psi-1$ & $\tau_{n}$ & $\tau_{k}$ & $\tau_{c}$ & $\frac{\mathrm{m}}{\mathrm{y}}$ & $\frac{\mathrm{g}}{\mathrm{y}}$ & $\frac{\mathrm{b}}{\mathrm{y}}$ & $\mathrm{R}_{b}$ & $\mathrm{n}$ \\
\hline $\begin{array}{c}\text { Tasa de } \\
\text { crecimiento } \\
\text { per cápita }\end{array}$ & $\begin{array}{c}\text { Impuesto } \\
\text { al trabajo }\end{array}$ & $\begin{array}{c}\text { Impuesto } \\
\text { al capital }\end{array}$ & $\begin{array}{c}\text { Impuesto } \\
\text { al } \\
\text { consumo }\end{array}$ & $\begin{array}{c}\text { Importaciones } \\
\text { netas/PIB }\end{array}$ & $\begin{array}{c}\text { Consumo de } \\
\text { gobierno } \\
\text { /PIB }\end{array}$ & $\begin{array}{c}\text { Deuda } \\
\text { /PIB }\end{array}$ & $\begin{array}{c}\text { Tasa de } \\
\text { interés } \\
\text { de largo } \\
\text { plazo }\end{array}$ & $\begin{array}{c}\text { Horas de } \\
\text { trabajo }\end{array}$ \\
\hline
\end{tabular}

\subsection{Tasas impositivas efectivas}

Las tasas impositivas marginales estatutarias, es decir, las que se reflejan en las leyes correspondientes, son en general distintas de las tasas impositivas efectivamente realizadas, debido entre otras cosas a evasión y elusión fiscal, fallas en la administración tributaria, deducciones, así como a regímenes y tratamientos especiales. Las tasas efectivas calculadas con la metodología de Mendoza et al. (1994) son una buena estimación del efecto que tienen las tasas impositivas estatutarias sobre la economía agregada, ver Uhlig y Trabandt (2009).

El método de Mendoza et al. (1994) da como resultado aproximaciones útiles para modelos de equilibrio general dinámicos con agente representativo de las tasas impositivas al trabajo, a los ingresos al capital y al consumo. Dicho procedimiento permite calcular series de tiempo de tasas impositivas efectivas al consumo, a los ingresos al capital y a los ingresos laborales utilizando información publicada por la OCDE.

El método utilizado produce tasas impositivas efectivas agregadas, que de hecho corresponden a las tasas impositivas que en promedio ocurren a nivel macro. Estas tasas capturan la información agregada sobre las tasas establecidas, los créditos, las deducciones y las exenciones implícitas en las cuentas nacionales y estadísticas recaudatorias de modo que capturan la carga impositiva general de cada impuesto y mantienen la consistencia con el marco de agente representativo. La tasa efectiva es el cociente de la recaudación de un tipo de impuesto dividida por la base impositiva potencial para ese impuesto.

\subsection{Fórmulas para el cálculo de las tasas impositivas efectivas}

La OCDE provee de forma pública agregados de impuestos de acuerdo a una clasificación propia. Ésta separa la recaudación impositiva principalmente por la base sobre la que se levanta el gravamen, empleando códigos numéricos de cuatro dígitos para distinguirlas. Cuando el código se encuentra seguido de tres ceros se refiere a la clasificación principal, mientras que variaciones en los tres últimos dígitos indican sub-clasificaciones.

La recaudación impositiva por tipo de base se clasifica de la siguiente manera: ingreso, utilidades y ganancias por capital (1000); nómina y fuerza de trabajo (3000); propiedad (4000) y bienes y servicios (5000), entre otros. La OCDE también considera como parte de los impuestos las contribuciones a seguridad social (2000). Esta desagregación permite estimar las tasas impositivas efectivas al consumo, a los ingresos laborales y a los ingresos al capital. A continuación se presentan las fórmulas empleadas en la 
metodología de Mendoza et al. (1994):

$$
\begin{gathered}
\text { Impuesto al consumo: } \tau^{\mathrm{c}}=\frac{5110+5121}{\mathrm{C}+\mathrm{G}-\mathrm{GW}-5110-5121} \\
\text { Impuesto al ingreso personal: } \tau^{\mathrm{h}}=\frac{1100}{\mathrm{OSPUE}+\mathrm{PEI}+\mathrm{W}} \\
\text { Impuesto al ingreso laboral: } \tau^{\mathrm{n}}=\frac{\tau^{\mathrm{h}} \mathrm{W}+2000+3000}{\mathrm{~W}+2200} \\
\text { Impuesto a los ingresos al capital: } \tau^{\mathrm{k}}=\frac{\tau^{\mathrm{h}}(\text { OSPUE }+ \text { PEI })+1200+4100+4400}{\mathrm{OS}}
\end{gathered}
$$

Donde $C$ es consumo privado nominal total, $G$ es el consumo de gobierno, $W$ son salarios y sueldos de la economía, $G W$ es compensación a los empleados (gobierno general), OSPUE son los ingresos operativos de las empresas privadas no constituidas en sociedad, $P E I$ son las rentas netas por propiedades y $O S$ son los ingresos operativos netos de la economía.

\subsection{Estimación de tasas impositivas efectivas}

El presente estudio no es el primero en estimar las tasas efectivas al consumo, a los ingresos laborales y al capital para México. Existen estudios previos como es el caso de Dalsgaard (2000) y Antón (2005) que estiman estas tasas efectivas con el método de Mendoza et al. (1994) y otras extensiones, ambos realizan los cálculos respectivos con datos proporcionados por la OCDE.

Como se mostró en la primera parte la recaudación tributaria como porcentaje del PIB en México presenta los niveles más bajos entre los países de la OCDE, este es el caso aun tomando en cuenta los recursos provenientes de fuentes petroleras. Asimismo los recursos provenientes del petróleo son parte importante de la recaudación tributaria, alrededor de 30\% de los ingresos del gobierno federal son petroleros. ${ }^{2}$ Es por estas razones que se debería esperar que las tasas impositivas efectivas sean pequeñas en relación con las de otros países.

Mientras que el presente documento estima las tasas efectivas mencionadas, para México de 2003 a 2010, Dalsgaard (2000) cubre el periodo de 1980 a 1996 y Antón (2005) el de 1993 a 2001. Dalsgaard (2000) estima las tasas respectivas empleando una versión modificada del método de Mendoza et al. (1994). Antón (2005) estima dichas tasas con el método de Mendoza et al. (1994) y adicionalmente emplea extensiones con los métodos de Carey y Tchilinguirian (2000) y Carey y Rabesona (2002).

\footnotetext{
2 La recaudación estimada para los tres impuestos es menor a la recaudación tributaria que registra la OCDE para México pues no toma en cuenta los derechos a los hidrocarburos.
} 
Tabla 2. Tasas impositivas efectivas promedio, México

\begin{tabular}{|c|c|c|c|}
\hline \multicolumn{4}{|c|}{ Porcentaje } \\
\hline & Dalsgaard (2000) & Antón (2005) & Presente estudio \\
\hline & $1980-1996$ & $1993-2001$ & $2003-2010$ \\
\hline Trabajo & 11.5 & $8.0-12.6$ & 13.6 \\
\hline Capital & 5.6 & $8.5-15.1$ & 6.5 \\
\hline Consumo & 13.8 & $6.9-13.7$ & 6.7 \\
\hline
\end{tabular}

Fuente:Elaboración propia.

A continuación se presentan las estimaciones de las tasas efectivas y la recaudación asociada como porcentaje del PIB, el periodo de análisis tanto para México como para Estados Unidos va de 2003 a 2010.

Gráfico 2. Impuesto al trabajo, tasa efectiva



Gráfico 3. Impuesto al capital, tasa efectiva

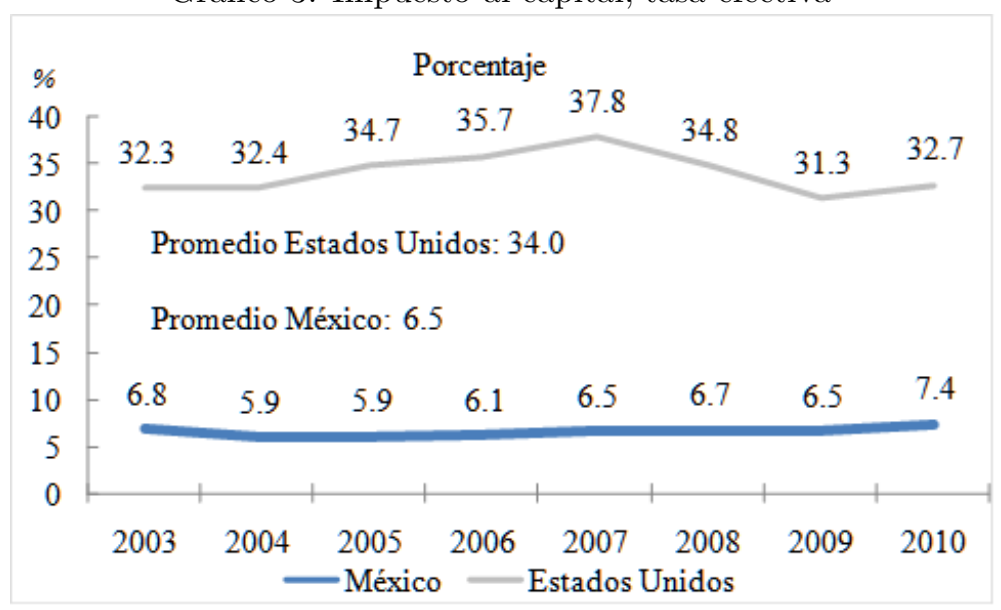


Gráfico 4. Impuesto al consumo, tasa efectiva. Porcentaje

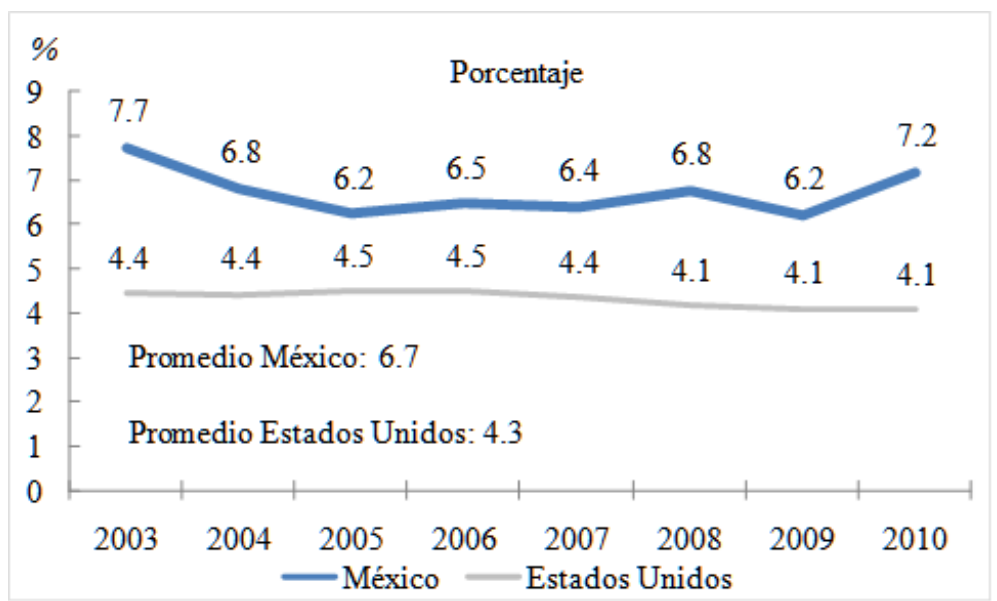

\section{Curvas de Laffer: México y Estados Unidos}

Cada una de las curvas de Laffer se estima variando un impuesto y manteniendo constantes los demás parámetros, incluyendo los valores para los otros dos impuestos. Llamaremos recaudación base a aquella que se estima empleando el promedio de las variables mencionadas anteriormente durante el periodo de 2003 a 2010 tanto para Estados Unidos como para México, así como con el valor elegido para los distintos parámetros. La recaudación base es una aproximación de la recaudación impositiva del país en cuestión para ese periodo; la recaudación se indiza a 100 en el escenario base. El que la curva de Laffer de un país esté por encima de la del otro no significa que la recaudación pueda ser mayor, sino que el crecimiento con respecto a la recaudación base puede ser mayor.

Las diferencias en las estimaciones entre México y Estados Unidos surgen principalmente de las disparidades en la recaudación efectiva medida a través de las tasas impositivas efectivas. La tasa promedio del impuesto al trabajo en México (13.6\%) para el periodo de análisis es prácticamente la mitad que la de Estados Unidos (26.3\%) mientras que la tasa impositiva a los ingresos al capital en Estados Unidos (34.0\%) es cinco veces más grande que en México (6.5\%). En contraste, en el impuesto al consumo la diferencia no es tan marcada, la tasa efectiva para Estados Unidos (4.3\%) es la tercera parte de la de México $(6.7 \%)$.

Tabla 3. Variables para calibrar: México y Estados Unidos

\begin{tabular}{|l|c|c|c|c|c|c|c|c|c|}
\hline & $\psi-1$ & $\tau_{n}$ & $\tau_{k}$ & $\tau_{c}$ & $\frac{\mathrm{m}}{\mathrm{y}}$ & $\frac{\mathrm{g}}{\mathrm{y}}$ & $\frac{\mathrm{b}}{\mathrm{y}}$ & $\mathrm{R}_{b}$ & $\mathrm{n}$ \\
\hline & $\begin{array}{c}\text { Tasa de } \\
\text { crecimiento } \\
\text { per cápita }\end{array}$ & $\begin{array}{c}\text { Impuesto } \\
\text { al trabajo }\end{array}$ & $\begin{array}{l}\text { Impuesto } \\
\text { al capital }\end{array}$ & $\begin{array}{c}\text { Impuesto } \\
\text { al } \\
\text { consumo }\end{array}$ & $\begin{array}{c}\text { Importaciones } \\
\text { netas/PIB }\end{array}$ & $\begin{array}{c}\text { Consumo } \\
\text { de } \\
\text { gobierno } \\
\text { /PIB }\end{array}$ & $\begin{array}{c}\text { Deuda } \\
\text { /PIB }\end{array}$ & $\begin{array}{c}\text { Tasa } \\
\text { de } \\
\text { interés }\end{array}$ & $\begin{array}{c}\text { Horas } \\
\text { de } \\
\text { trabajo }\end{array}$ \\
\hline México & 6.7 & 13.6 & 6.5 & 6.7 & 1.6 & 13.5 & 23.1 & 7.3 & 26.1 \\
\hline $\begin{array}{l}\text { Estados } \\
\text { Unidos }\end{array}$ & 3.0 & 26.3 & 34.0 & 4.3 & 4.7 & 18.9 & 41.7 & 4.0 & 23.8 \\
\hline
\end{tabular}


Dados los parámetros, se encuentran las curvas de Laffer para México y Estados Unidos para el impuesto al trabajo, el impuesto al consumo y a los ingresos al capital. A continuación se presentan las estimaciones de dichas curvas.

\subsection{Curvas de Laffer en el impuesto al trabajo}

Como se puede observar en la Gráfica 5, variando únicamente el impuesto al trabajo, la recaudación puede crecer casi cuatro veces más en México que en Estados Unidos, donde la tasa del impuesto al trabajo que maximiza la recaudación es mayor para México.

Asimismo, la pendiente de la curva de Laffer en el impuesto al trabajo para Estados Unidos es más baja que la de México. Esto se debe a que dado que las tasas efectivas al capital son mucho más altas en Estados Unidos, la economía es más sensible a cambios en la tasa impositiva al trabajo.

Para México, el valor promedio para la tasa efectiva del impuesto al trabajo es de $13.6 \%$, el nivel que maximiza la recaudación manteniendo todo lo demás constante es de aproximadamente $60 \%$ alcanzando un nivel que es $96 \%$ superior a la recaudación base, ver Gráfica 5. Para Estados Unidos, el valor para la tasa efectiva del impuesto al trabajo es de $26.3 \%$, el nivel que maximiza la recaudación manteniendo todo lo demás constante es de aproximadamente $55 \%$ alcanzando un nivel que es $24 \%$ superior a la recaudación base, ver Gráfica 5 .

Gráfico 5. Curva de Laffer para el impuesto al trabajo: México y Estados Unidos

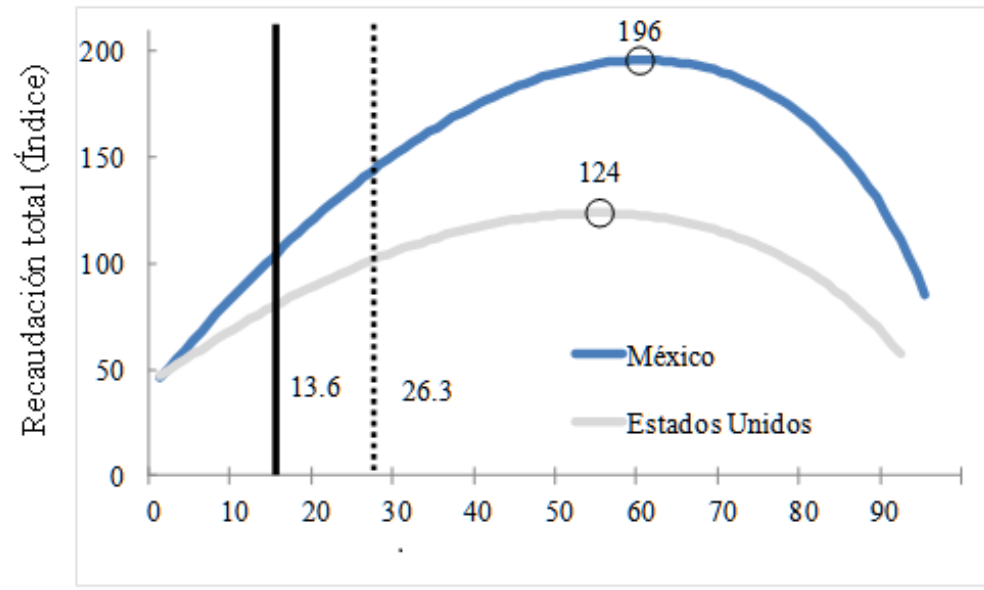

Tasa del impuesto al trabajo (\%)

Nota: la línea sólida indica la tasa impositiva promedio al trabajo en dicho periodo para México, la línea punteada corresponde a Estados Unidos. Los círculos señalan los niveles máximos en cada curva.

\subsection{Curvas de Laffer en el impuesto al capital}

Como se puede observar en la Gráfica 6, variando únicamente el impuesto al capital, la recaudación puede crecer aproximadamente doce veces más en México 
que en Estados Unidos, donde la tasa del impuesto al capital que maximiza es mayor para México. Las curvas de Laffer en el impuesto al capital para ambos países son más planas que las curvas correspondientes al impuesto al trabajo pues la economía es más sensible a cambios en el impuesto a los ingresos al capital.

Además, la pendiente de la curva de Laffer en el impuesto al capital para Estados Unidos es en general más baja, dado que los impuestos al trabajo son más altos en este país, la economía es más sensible a cambios en el impuesto al capital. Para México, el valor promedio para la tasa efectiva del impuesto al capital es de $6.5 \%$, el nivel que maximiza la recaudación manteniendo todo lo demás constante es de aproximadamente 58\% alcanzando un nivel que es $37 \%$ superior a la recaudación base, ver Gráfica 6 .

Para Estados Unidos, el valor promedio para la tasa efectiva del impuesto al capital es de $34 \%$, el nivel que maximiza la recaudación manteniendo todo lo demás constante es de $52 \%$ alcanzando un nivel que es $2.6 \%$ superior a la recaudación base, ver Gráfica 6 .

Gráfico 6. Curva de Laffer para el impuesto al capital: México y Estados Unidos

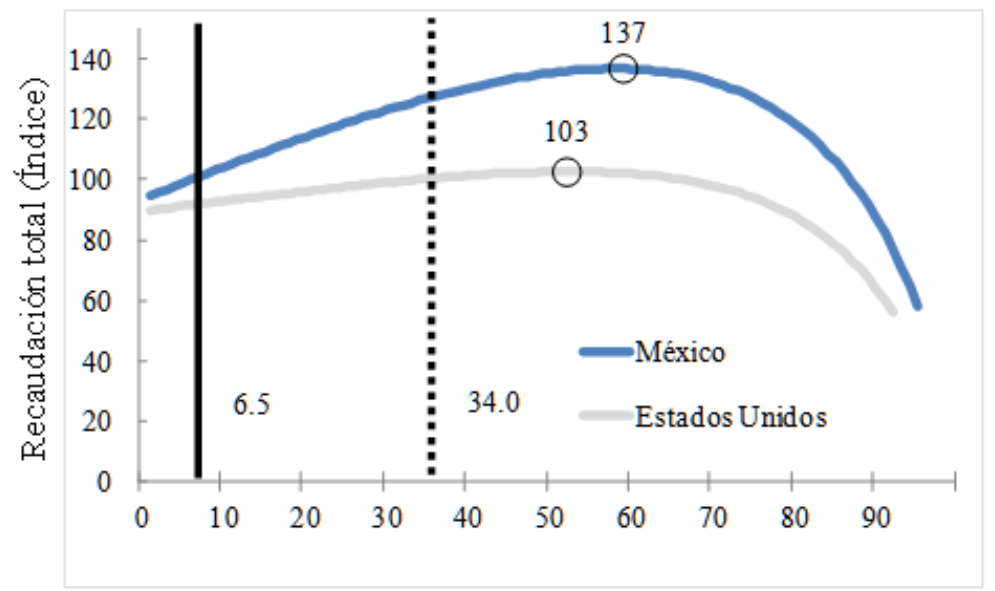

Tasa del impuesto al capital (\%)

Nota: la línea sólida indica la tasa impositiva promedio al capital en dicho periodo para México, la línea punteada corresponde a Estados Unidos. Los círculos señalan los niveles máximos en cada curva.

\subsection{Curvas de Laffer en el impuesto al consumo}

La curva de Laffer en el impuesto al consumo es creciente a tasas decrecientes y tiende a converger a un valor finito, ver Uhlig y Trabandt (2009). Esto se debe a que la recaudación adicional se emplea en transferencias de suma fija que se destinan a los hogares, las cuales de emplearse en consumo están gravadas. Aunque las tasas impositivas promedio al consumo no son muy distintas, México puede aumentar más la recaudación incrementando la tasa del impuesto al 
consumo pues el nivel en los demás impuestos es relativamente menor al de Estados Unidos.

Para México, el valor promedio para el escenario base de la tasa efectiva del impuesto al consumo es de $6.7 \%$. Elevando la tasa a $50 \%$ la recaudación puede aumentar en 140\%, mientras que para un nivel de $95 \%$ ésta puede crecer aproximadamente $258 \%$ con respecto a la recaudación base, ver Gráfica 7 .

Para Estados Unidos, el valor promedio para el escenario base de la tasa efectiva del impuesto al consumo es de $4.3 \%$. Elevando la tasa a $50 \%$ la recaudación puede aumentar en $107 \%$, mientras que para un nivel de $95 \%$ ésta puede crecer aproximadamente $194 \%$ con respecto a la recaudación base, ver Gráfica 7.

Gráfica 7. Curva de Laffer para el impuesto al consumo:

México y Estados Unidos

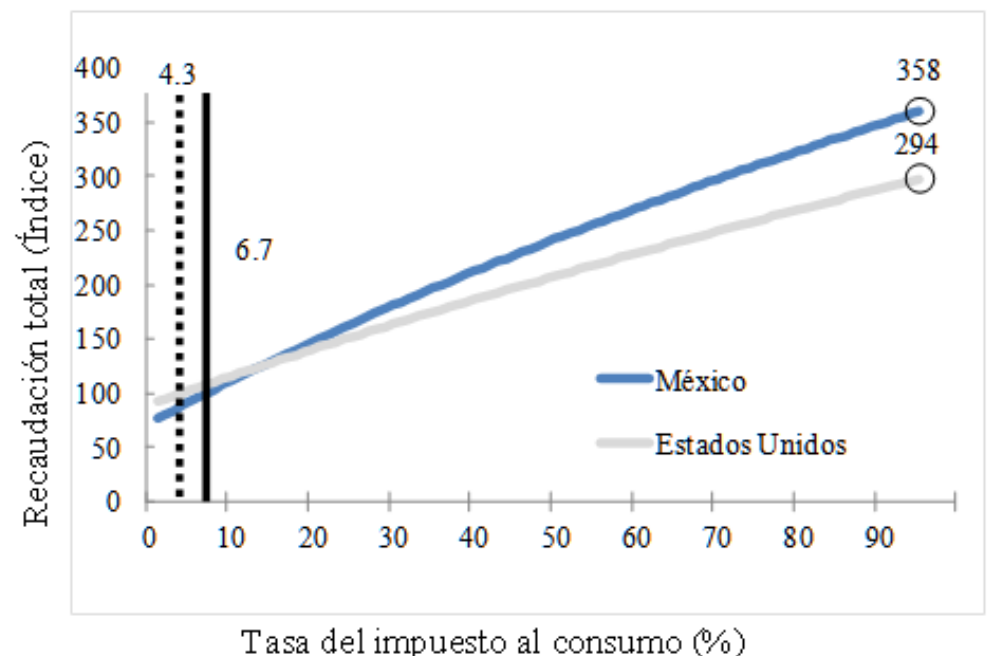

Nota: la línea sólida indica la tasa impositiva promedio al consumo en dicho periodo para México, la línea punteada corresponde a Estados Unidos. Los círculos señalan los niveles máximos en cada curva.

\section{Montaña de Laffer en el impuesto al trabajo y a los ingresos al capital}

La montaña de Laffer en el impuesto al trabajo y a los ingresos al capital representa el conjunto de valores que la recaudación total puede tomar al variar de manera conjunta los dos impuestos mencionados, manteniendo constante el impuesto al consumo. La montaña, por ende, se encuentra conformada por el conjunto de curvas de Laffer en uno de los impuestos para cada nivel del otro impuesto.

Tanto México como Estados Unidos se encuentran en el lado incorrecto de la montaña con respecto al capital, ver Gráficas 8 y 9 . Esto quiere decir que se encuentran en el rango normal para el impuesto al trabajo y en el prohibitivo para el impuesto al capital. Como se vio anteriormente esto significa que ambos 
pueden mantener su recaudación disminuyendo las tasas efectivas al capital y al trabajo en una cierta proporción. También se implica que, de aumentar la tasa efectiva al trabajo se obtiene una recaudación mucho mayor disminuyendo la tasa al capital que aumentándola; de este modo se promueve la acumulación de capital y se compensa el aumento en el otro impuesto, lo que hace que aumente la recaudación.

En cuanto al máximo recaudatorio las estimaciones obtenidas indican que, llevando a $5 \%$ la tasa del impuesto al capital y a alrededor de $60 \%$ la tasa del impuesto al trabajo la recaudación puede aumentar para México aproximadamente $100 \%$ con respecto a la situación base como se muestra en la Gráfica 8, con una combinación análoga Estados Unidos podría aumentarla $26 \%$, como se observa en la Gráfica 9. De disminuir más la tasa del impuesto al capital, a niveles cercanos a cero, los resultados no cambian visiblemente en comparación con tasas del $5 \%$.

Como se puede observar, comparando la Gráfica 8 con la 9, la montaña de Laffer para México es más alta que para Estados Unidos, esto no implica que la recaudación en México pueda ser mayor a la de Estados Unidos, sino que como proporción de la recaudación base puede crecer más que la de Estados Unidos. Se puede apreciar sin embargo que Estados Unidos se encuentra más cerca del máximo recaudatorio con respecto a ambos impuestos, esto se debe a que, como se mencionó anteriormente, ambas tasas efectivas promedio son mucho mayores que en México.

Gráfica 8. Montaña de Laffer: México

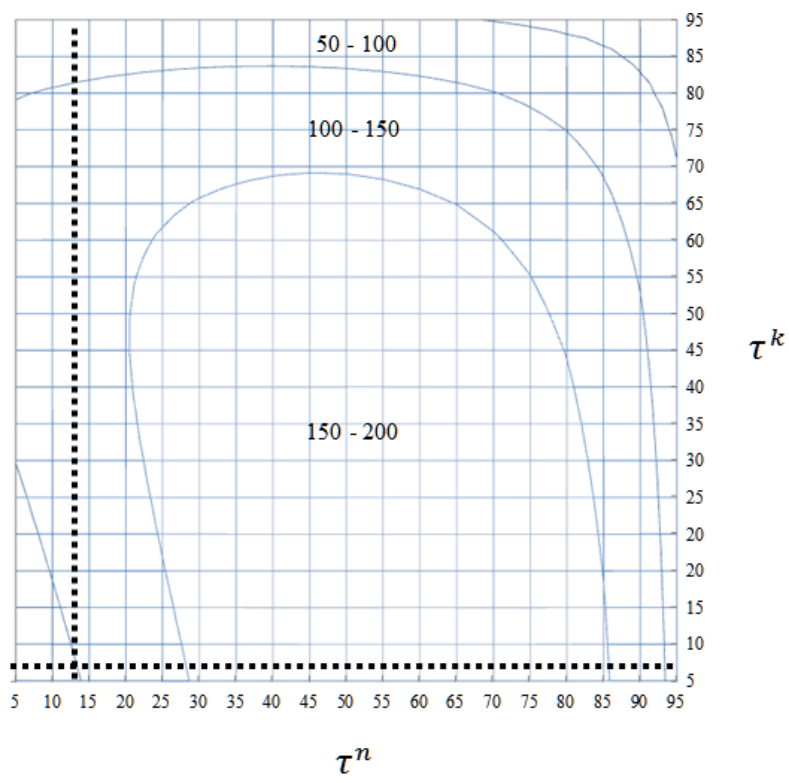


190 Nueva Época REMEF (The Mexican Journal of Economics and Finance)

Gráfica 8. Montaña de Laffer: México (continuación)

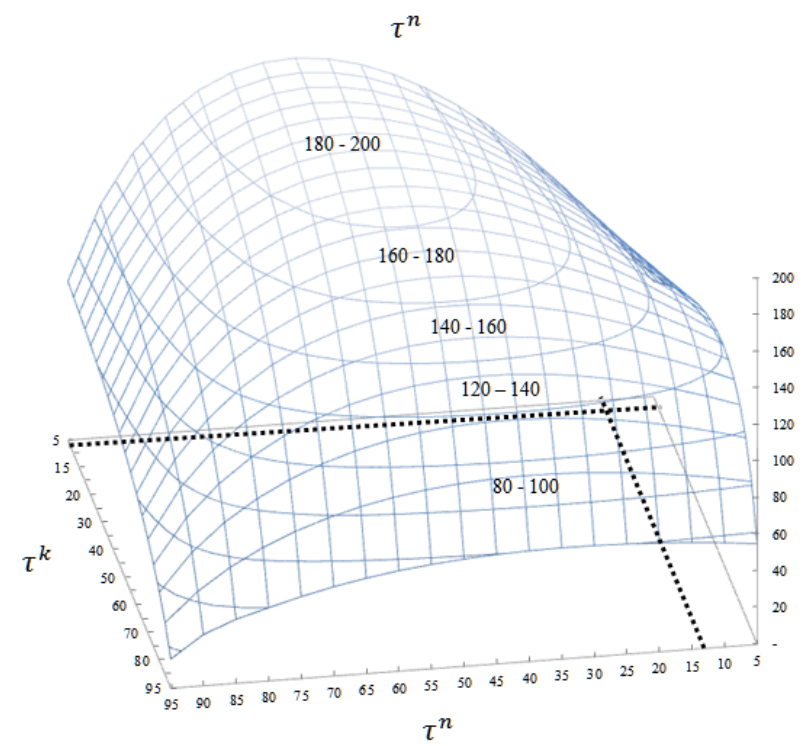

Nota: las líneas punteadas señalan el nivel promedio de la tasa efectiva en el periodo de estudio. Las tasas se expresan en porcentaje.

Gráfica 9. Montaña de Laffer: Estados Unidos

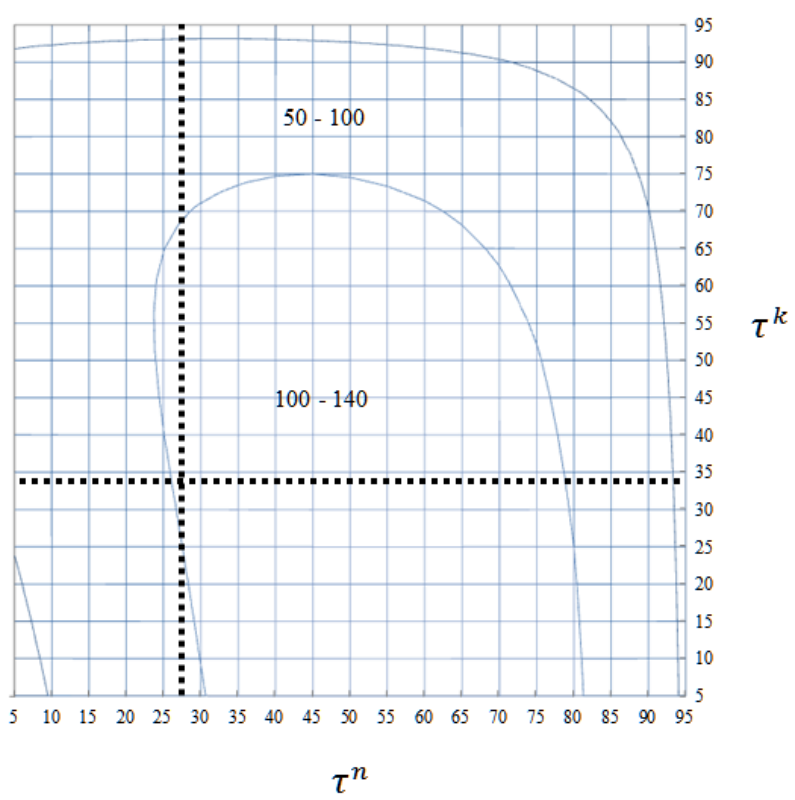


Gráfica 9. Montaña de Laffer: Estados Unidos (continuación)

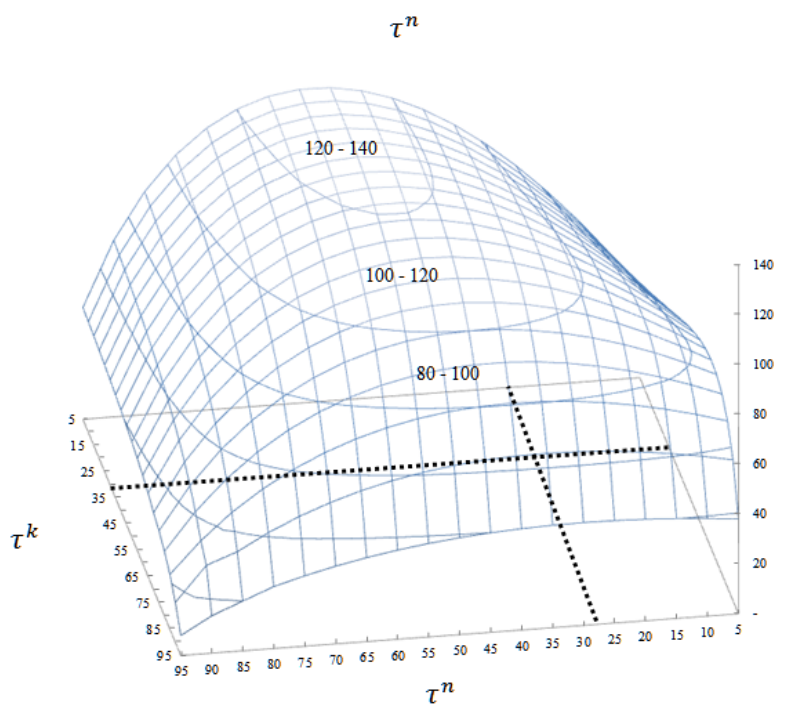

Nota: las líneas punteadas señalan el nivel promedio de la tasa efectiva en el periodo de estudio. Las tasas se expresan en porcentaje.

\section{Resumen de estimaciones obtenidas}

A continuación se presenta un resumen de las estimaciones obtenidas a partir del análisis de las curvas de Laffer y la montaña de Laffer en el impuesto al trabajo y a los ingresos al capital. Donde $\tau_{0}$ se refiere a la tasa impositiva efectiva de un impuesto particular en el periodo base, $\tau_{*}$ representa la tasa que maximiza la recaudación, ya sea a lo largo de una curva particular o con relación a la montaña y $T_{*}$ es la recaudación adicional como proporción de la recaudación base que se obtendría de maximizar a lo largo de una curva o con relación a la montaña. El súper índice indica de qué impuesto se trata, $n$ para el impuesto al trabajo y $k$ para el impuesto a los ingresos al capital.

Tabla 4. Tasas impositivas efectivas

\begin{tabular}{|c|c|c|}
\hline \multicolumn{3}{|c|}{ Porcentaje } \\
\hline & $\tau^{n}$ & $\tau^{k}$ \\
\hline México & 13.6 & 6.5 \\
\hline Estados Unidos & 26.3 & 34.0 \\
\hline
\end{tabular}

Tabla 5. Curvas de Laffer, México

\begin{tabular}{|c|c|c|c|}
\hline \multicolumn{4}{|c|}{ Porcentaje } \\
\hline & $\tau_{0}$ & $\tau_{*}$ & $T_{*}$ \\
\hline Impuesto al trabajo & 13.6 & 60.0 & 95.8 \\
\hline Impuesto al capital & 6.5 & 58.0 & 36.7 \\
\hline
\end{tabular}


Tabla 6. Curvas de Laffer, Estados Unidos

\begin{tabular}{|c|c|c|c|}
\hline \multicolumn{4}{|c|}{ Porcentaje } \\
\hline & $\tau_{0}$ & $\tau_{*}$ & $T_{*}$ \\
\hline Impuesto al trabajo & 26.3 & 55.0 & 23.7 \\
\hline Impuesto al capital & 34.0 & 52.0 & 2.6 \\
\hline
\end{tabular}

Tabla 7. Montañas de Laffer, México y Estados Unidos

\begin{tabular}{|c|c|c|c|}
\hline \multicolumn{4}{|c|}{ Porcentaje } \\
\hline & $\tau_{*}^{n}$ & $\tau_{*}^{k}$ & $T_{*}$ \\
\hline Impuesto al trabajo & 60.0 & 5.0 & 99.8 \\
\hline Impuesto al capital & 60.0 & 5.0 & 26.2 \\
\hline
\end{tabular}

\section{Conclusiones}

En comparación con Estados Unidos, las tasas efectivas de México son muy bajas. La tasa promedio del impuesto al trabajo en México (13.6\%) en el periodo de análisis, de 2003 a 2010, es prácticamente la mitad que la de Estados Unidos $(26.3 \%)$ mientras que la tasa impositiva a los ingresos al capital en Estados Unidos (34.0\%) es cinco veces más grande que en México (6.5\%). En el impuesto al consumo sin embargo, la tasa efectiva para Estados Unidos (4.3\%) es la tercera parte que la de México $(6.7 \%)$.

En términos de las curvas de Laffer para el impuesto al trabajo y al capital, Estados Unidos puede aumentar su recaudación en $24 \%$ aumentando al máximo la tasa efectiva al trabajo (de $26.3 \%$ a $55 \%$ ) y en $3 \%$ aumentando al máximo la tasa efectiva a los ingresos al capital (de $34 \%$ a $52 \%$ ). México puede aumentar su recaudación en $96 \%$, casi al doble, aumentando al máximo la tasa efectiva al trabajo (de $13.6 \%$ a $60 \%$ ) y $37 \%$ aumentando al máximo la tasa efectiva a los ingresos al capital (de 6.5\% a 58\%). El impuesto al consumo no presenta una curva de Laffer, sin embargo, la recaudación es creciente en este impuesto y converge a un valor finito.

Por lo tanto, con el presente documento, que constituye una extensión a Uhlig y Trabandt (2009), se encuentra que México no puede autofinanciar un recorte de impuestos ni en el impuesto al trabajo ni en el que grava al capital, ya que se encuentra en el tramo normal para ambos impuestos por separado.

Uhlig y Trabandt (2009), con un modelo igual al del presente documento encuentran que Suecia y Dinamarca sí están en el lado prohibitivo de la curva de Laffer para el impuesto a los ingresos al capital, por lo que pueden autofinanciar un recorte en este impuesto. Para el impuesto al trabajo, todos los países analizados en dicho estudio ${ }^{3}$ se encuentran en el lado normal de la curva de Laffer.

Para observar la respuesta de la recaudación agregada ante variaciones conjuntas de ambos impuestos, se estimó una montaña de Laffer para Estados

\footnotetext{
3 Alemania, Francia, Italia, Reino Unido, Austria, Bélgica, Dinamarca, Finlandia, Grecia, Irlanda, Holanda, Portugal, España y Suecia.
} 
Unidos y México. La montaña de Laffer describe la relación que existe entre la recaudación agregada y distintas combinaciones de impuestos al trabajo y al capital.

En términos de la montaña de Laffer tanto Estados Unidos como México se encuentran en el lado prohibitivo con respecto al impuesto al capital y en el tramo normal con respecto al impuesto al trabajo. Esto significa que pueden aumentar más su recaudación en el largo plazo disminuyendo el impuesto al capital y aumentando el impuesto al trabajo que elevando ambos. Uhlig y Trabandt (2009) muestran que esto también se cumple tanto para Estados Unidos como para la Unión Europea tomando de forma agregada a catorce países.

En cuanto al máximo recaudatorio en términos de la montaña de Laffer, las estimaciones obtenidas indican que llevando a $5 \%$ la tasa del impuesto al capital y a $60 \%$ la tasa del impuesto al trabajo, la recaudación puede aumentar para México aproximadamente $100 \%$ con respecto a la situación base, mientras que Estados Unidos podría aumentarla $26 \%$ con una combinación análoga.

Aunque los resultados de este estudio arrojen que tanto Estados Unidos como México se encuentran en posibilidades de aumentar la recaudación mediante alzas en las tasas impositivas eso no significa que deban aumentarlas. De este documento se desprende que si fuera necesario aumentar la recaudación esto se debe llevar a cabo preferentemente a través de incrementar las tasas efectivas y no las estatutarias, es decir aumentando la recaudación efectiva. ${ }^{4}$

El nivel que deban tener los impuestos depende del nivel de gasto público requerido. Una extensión interesante a este documento sería evaluar qué punto de la montaña de Laffer es el óptimo social para México, considerando que los impuestos pueden tener tanto efectos negativos en términos de eficiencia como efectos positivos sobre el bienestar social a través del gasto que financian, redistribuyendo la riqueza así cómo proporcionando bienes y servicios públicos.

El presente análisis ayuda a tener una estimación de en qué posición se encuentra la economía mexicana con respecto a la recaudación potencial. Esto es útil en términos de política fiscal, pues mientras más cerca se esté del máximo, mayores son las pérdidas en eficiencia asociadas a un aumento en las tasas impositivas comparadas con las ganancias en recaudación.

\section{Bibliografía}

Antón, A. (2005). Average effective Tax Rates in Mexico. Economía Mexicana Nueva Época, $\mathrm{XIV}(2)$, segundo semestre.

Carey, D. y J. Rabesona. (2002). Tax Ratios on Labour and Capital Income and Consumption. OECD Economic Studies, 35, pp. 129-74.

\footnotetext{
4 De acuerdo con Dalsgaard (2000) para aumentar la recaudación efectiva en México hay que mejorar la recolección y aumentar la base impositiva de los distintos impuestos. Para aumentar la base impositiva en México, un camino viable es disminuir los regímenes especiales, los tratos preferenciales reducen la recaudación y complican la administración tributaria, ver Dalsgaard (2000). Los tratos preferenciales generan lagunas fiscales que bien pueden ser aprovechadas por los contribuyentes para llevar a cabo una planeación fiscal efectiva y facilitar la evasión fiscal.
} 
194 Nueva Época REMEF (The Mexican Journal of Economics and Finance)

Carey, D. y H. Tchilinguirian. (2000). Average Effective Tax Rates on Capital, Labour and Consumption. OECD Economics Department, Working Papers No.258, OECD Publishing.

Dalsgaard, T. (2000). The Tax System in Mexico: A Need for Strengthening the RevenueRaising Capacity. OECD Economics Department, OECD Publishing.

Frenkel, J. A. Razin y E. Sadka. (1991). International Taxation in an Integrated World. Cambridge, Mass: MIT Press.

Fullerton, D. (1980). On the Possibility of an Inverse Relationship between Tax Rates and Government Revenues. National Bureau of Economic Research, Working Paper No. 467, New York.

Laffer, A. (1981). Government Exactions and Revenue Deficiencies. Cato Journal, 1, Cato Institute.

Mendoza, E. G., A. Razin y L.L. Tesar. (1994). Effective Tax Rates in Macroeconomics: Cross-country estimates of Tax Rates on Factor Incomes and Consumption. Journal of Monetary Economics, 34, pp. 297-323.

OCDE (Organización para la Cooperación y el Desarrollo Económico). (2007). Glossary of Statistical Terms. OECD Publishing.

OCDE (Organización para la Cooperación y el Desarrollo Económico). (2010). Revenue Statistics 2010 - Special feature: Environmental Related Taxation. OECD Publishing.

OCDE (Organización para la Cooperación y el Desarrollo Económico). (2011). Revenue Statistics in Latin America. OECD Publishing.

Ragan, K. (2005). Taxes, Transfers and Time Use: Fiscal Policy in a Household Production Model. University of Chicago.

Romer, C. y D. Romer. (2007). The Macroeconomic Effects of Tax Changes: Estimates Based on a New Measure of Fiscal Shocks. University of California, Berkley.

SHCP (Secretaría de Hacienda y Crédito Público). (2011a). El Impuesto Empresarial a Tasa nica (IETU): Un diagnóstico a tres años de su implementación. México.

SHCP (Secretaría de Hacienda y Crédito Público). (2011b). Ley de Ingresos de la Federación 2012. México.

Uhlig, H. y M. Trabandt. (2009). How Far Are We from the Slippery Slope? The Laffer Curve Revisited. National Bureau of Economic Research, Working paper 15343, Cambridge. 